\title{
Temporal Analysis of GDOP to Quantify the Benefits of GPS and GLONASS Combination on Satellite Geometry
}

\author{
Claudio Meneghini ${ }^{1}$, Claudio Parente ${ }^{2}$ \\ Department of Sciences and Technologies \\ University of Naples "Parthenope" \\ Naples, Italy
}

\begin{abstract}
Global Navigation Satellite Systems (GNSS) have developed rapidly over the last few years. At present, there are GNSS receivers that combine satellites from two or more different constellations. The geometry of the satellites in relation to the receiver location, i.e. how nearly or distantly they are disposed in the sky, impacts on the quality of the survey, which is essential to achieve the highest level of position accuracy. A dimensionless number identified as Geometric Dilution of Precision (GDOP) is used to represent the efficiency of the satellite distribution and can be easy calculated for each location and time using satellite ephemeris. This paper quantifies the influence of multi-GNSS constellation, in particular GPS (Global Positioning System) and GLONASS (Globalnaya Navigazionnaya Sputnikovaya Sistema) combination, on satellite geometry considering a precise period. A new index named Temporal Variability of Geometric Dilution of Precision (TVGDOP) is proposed and analyzed in different scenarios (different cut-off angles as well as real obstacles such as terrain morphology and buildings). The new index is calculated for each of the two satellite systems (GPS and GLONASS) as well as for their integration. The TVGDOP values enable the three cases to be compared and permit to quantify the benefits of GNSS integration on satellite geometry. The results confirm the efficiency of the proposed index to highlight the better performance of combination GPS+GLONASS especially in presence of obstacles.
\end{abstract}

Keywords-GDOP (Geometric Dilution of Precision); GPS (Global Positioning System); GLONASS (Globalnaya Navigazionnaya Sputnikovaya Sistema); Multi-GNSS (Global Navigation Satellite System) Constellation

\section{INTRODUCTION}

Satellite constellations were designed to provide threedimensional navigation by determining the position of a receiver on land, at sea, or in space [1]. Errors in determining the position of receivers are caused by the precision that characterizes the measure of the distance to each satellite, and the placement of the satellites relatively to the receiver location, i.e. how nearly or distantly they are disposed in the sky [2].

Various techniques can be used to limit distance errors, but satellite geometry is essential to achieve the highest level of position accuracy. This geometry is often expressed using a numerical measure named "Dilution of Precision", or DOP. There are several types of DOPs, which are all functions of the receiver-transmitter geometry. The Geometric Dilution of Precision (GDOP) is a dimensionless quantity that represents the efficiency of the satellite distribution. If GDOP value is small, the position accuracy is high. With four satellites, which is the minimum number required for a single constellation, the best geometry is achieved when all four satellites together form a tetrahedron structure in which one of them is at the zenith and the others form an equilateral triangle. The value of the GDOP is small (so the accuracy is high), if the volume of the tetrahedron is large, as well as if the number of satellites is high [3]. The number of visible satellites influences positioning accuracy, availability and reliability: considering only one system such as GPS (Global Positioning System),, the visible satellites are often scarce in areas such as mountains, open-pit mines and urban canyons [4]. Sometimes satellite geometry can be inadequate even when four or more satellites of the same system are available. In such situations, the GNSS (Global Navigation Satellite Systems) multi-constellation approach is useful to increment the number of visible satellite, perform their geometry, and improve the continuity and reliability of the positioning.

Several studies and applications have demonstrated the benefits of multi-constellation operations in a receiver. Integrated GNSS significantly improves the results compared with them by each constellation, especially in obstructed areas [5] [6] [7] [8]. For example, combining GLONASS (Globalnaya Navigazionnaya Sputnikovaya Sistema) and GPS constellations, permits to achieve accessibility to a greater number of satellites in urban canyons, and better accuracy in zones of minimal availability [9].

Today more than 70 satellites are already in view, and about 120 satellites will be available once all four systems (BeiDou, Galileo, GLONASS and GPS) are fully deployed in the next few years [10].

The Global Positioning System (GPS) was the first global positioning system in the world and was created in 1973 by the US Department of Defense for military purposes. The first GPS satellite was launched in 1977, and the global positioning service reached full operational capacity 18 years later. Since 2000, GPS has been available free of charge for civilian purposes. At the time of writing, there are 31 operational satellites in the GPS constellation [11]. 
Developed by Russian Federal Space Agency in 1970, the GLONASS (Global'naya Navigatsionnaya Sputnikowaya Sistema) system was created for military purposes, and is managed by the Russian Ministry of Defense. The first GLONASS satellite was launched in 1984, and the GLONASS constellation reached the full working conditions of 24 satellites in 1996. Due to a lack of funds, the number of available satellites significantly decreased until December 2011 when the constellation again increased to 24 operating satellites. In 2006, GLONASS became free of charge for public usage. Currently there are 22 active satellites [12].

We performed a temporal analysis of the GDOP in order to evaluate the impacts of GPS and GLONASS combination on the quality of the GNSS survey. The GDOP was calculated as function of date and time considering three different constellations (GPS, GLONASS and GPS+GLONASS) and a period of 32 days. The variability of the cut-off angle as well as the presence of real obstacles were also considered. To facilitate a comparison of the various constellations and situations, a new index named TVGDOP (Temporal Variability of Geometric Dilution of Precision) produced as the inverse value of GDOP is introduced.

This paper is organized as follows. Section 2 presents the concepts of satellite geometry and Dilution of Precision (DOP). Section 3 describes and discusses the adopted approach. Finally, Section 4 draws some conclusions.

\section{THEORETICAL BACKGROUND}

\section{A. Satellite Geometry Impact on Positioning}

The geometric positions of the satellites in relation to the receiver can decrease navigation and survey accuracy. To explain this concept, it is worth looking at the case of two satellites. If the satellites are considered as the center, two arcs can be delineated from the satellites to represent positional lines. The first is internal with a radius equal to the true range, while the second is external with a pseudo-range as the radius.

The possible user position is defined by the intersection zone of the two arcs:

- If the distance between the satellites is significant (Fig. 1), the connection area is small. Consequently, a low position uncertainty means a good satellite geometry.

- If the satellites are near to each other (Fig. 2), the joint area is large. In this circumstance, considerable indecision regarding the position denotes a bad satellite geometry.

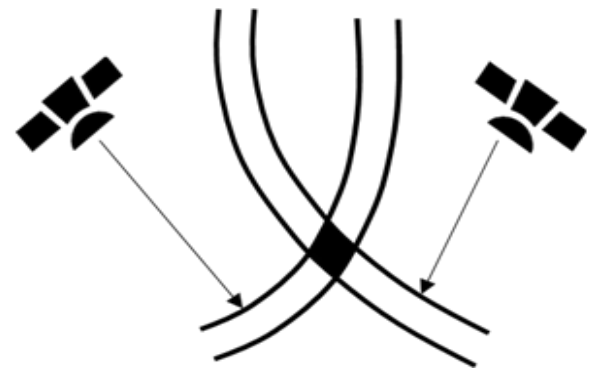

Fig. 1. A Low Position uncertainty for Two Satellites.

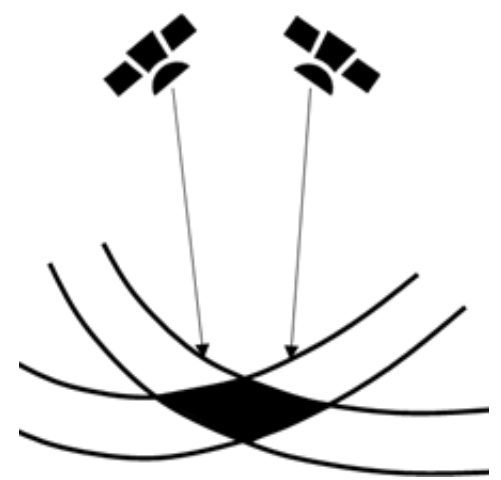

Fig. 2. A High uncertainty of Position for Two Satellites.
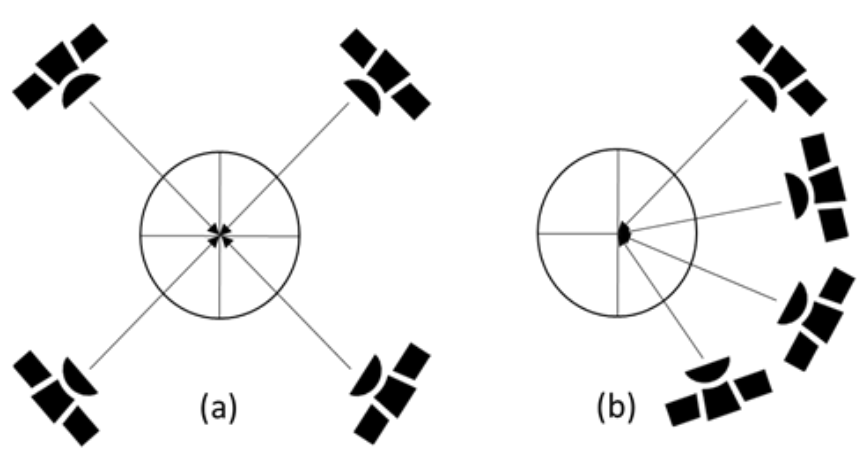

Fig. 3. Good (a) and Poor (b) Satellite Geometry.

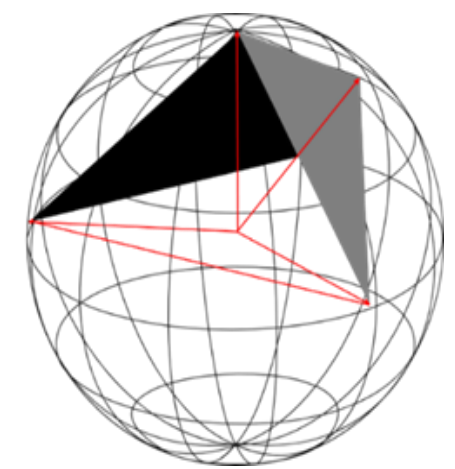

Fig. 4. The Tetrahedron Shaped by the Receiver and Four Satellites.

To fix the user position, at least four satellites (from the same constellation) are required. In this case, to gain a good geometry, all the available satellites must be distant from each other in the space. Fig. 3 represents a good and poor satellite geometry, respectively, with four satellites.

The best geometry with four satellites can be achieved if one of them is at the zenith and the others form an equilateral triangle. All the satellites together delimit a tetrahedron (Fig. 4).

If there are five or more satellites in view, only four of them, the ones corresponding to the best combination, are usually considered for redundancy reduction. To fix the receiver location, precise range measurements are necessary, more precisely, the measurements of ranges between the satellites and the receiver antenna. With four or more pseudorange observations, it is possible to calculate both the receiver's three-dimensional coordinates and its clock offset. 
The quality of the pseudo-range measurements defines the accuracy of the receiver coordinates. According to the literature [13] [14], the well-known equation to calculate the pseudo-range is:

$P=\rho+c(d T-d t)+d_{\text {ion }}+d_{\text {trop }}+e$

where:

- $\quad$ P represents the pseudo-range measurement;

- $\rho$ indicates the distance between the satellite antenna and the receiver antenna;

- $\mathrm{c}$ is the speed of light in a vacuum;

- $\mathrm{dT}$ is the receiver clock bias from the GNSS time;

- dt is the satellite clock bias from the GNSS time;

- $\mathrm{d}_{\text {ion }}$ is the ionospheric propagation delay;

- $\mathrm{d}_{\text {trop }}$ is the tropospheric propagation delay;

- $\mathrm{e}$ is the measurement noise in addition to multipath effect.

Considering that satellite clock bias, tropospheric delay and ionospheric delay are known, the pseudo-range equation becomes:

$P=\rho+c d T+e$

A receiver must resolve a number of equations equal to the number of measurements deriving from the visible satellites. At least four observations are needed to calculate the receiver coordinates.

The equations are not linear, but can be linearized considering the original estimates for the station's position. By correcting the initial estimates, it is possible to evaluate both the receiver's current coordinates and the clock offset. In addition, the equations can be grouped and represented in a matrix form:

$\delta P=A \delta U+n$

where:

- A is a matrix where each term defines the direction cosine vector from the receiver to the satellite;

- $\delta \mathrm{P}$ is a matrix of pseudorange observations;

- $\delta \mathrm{U}$ is a navigation error state vector including the receiver's position and clock bias;

- $\mathrm{n}$ is a vector containing the pseudo-range measurement noise.

The equation 3, with four visible satellites, takes the following form:

$\delta U=A^{-1} \delta P$

If there are five or more satellites, the receiver position is determined using the least squares method. In this case, the equation 3 can be rearranged as:

$\delta U=\left(A^{T} A\right)^{-1} A^{T} \delta P$ where:

- matrix A exemplifies the line of sight vectors that link the receiver to each satellite.

Assuming $\delta \mathrm{U}$ is a zero-mean vector that incloses the errors in the predicted operator state, the term $\delta U$ estimates the position error. Its covariance can be calculated using the generalized inverse of A [15]:

$\operatorname{cov}(\delta U)=E\left(\delta U \delta U^{T}\right)=$ $E\left[\left(A^{T} A\right)^{-1} A^{T} \delta P \delta P^{T} A\left(A^{T} A\right)^{-T}\right]=$

$\left(A^{T} A\right)^{-1} A^{T} d P d P^{T} A\left(A^{T} A\right)^{-T}=$

$\left(A^{T} A\right)^{-1} A^{T} \operatorname{cov}(\delta P) A\left(A^{T} A\right)^{-T}$

The term $\operatorname{cov}(\delta \mathrm{P})$ describes the pseudo-range errors, which being uncorrelated, are statistically independent. It follows that the covariance matrix is diagonal. If the variance $\left(\sigma_{n}\right)$ of the measurement errors for each satellite is the same, the term $\operatorname{cov}(\delta \mathrm{P})$ becomes:

$\operatorname{cov}(\delta P)=\sigma_{n}^{2}$

By substituting Eq. 7 into Eq. 6

$E\left(\delta U \delta U^{T}\right)=\sigma_{n}^{2}\left(A^{T} A\right)^{-1} A^{T} A\left(A^{T} A\right)^{-T}=\sigma_{n}^{2}\left(A^{T} A\right)^{-T}$

Under the assumption that $\left(A^{T} A\right)$ is symmetric, transpose is not necessary. Thus:

$\operatorname{cov}(\delta U)=\sigma_{n}^{2}\left(A^{T} A\right)^{-1}$

Supposingly $G=\left(A^{T} A\right)^{-1}$, it follows that:

$\operatorname{cov}(\delta U)=\sigma_{n}^{2} \mathrm{G}$

The covariance matrix is obtained by expanding the equation:

$\left[\begin{array}{cccc}\sigma_{x}^{2} & \operatorname{cov}(x, y) & \operatorname{cov}(x, z) & \operatorname{cov}(x, b) \\ \operatorname{cov}(y, x) & \sigma_{y}^{2} & \operatorname{cov}(y, z) & \operatorname{cov}(y, b) \\ \operatorname{cov}(z, x) & \operatorname{cov}(z, y) & \sigma_{z}^{2} & \operatorname{cov}(z, b) \\ \operatorname{cov}(b, x) & \operatorname{cov}(b, y) & \operatorname{cov}(b, z) & \sigma_{b}^{2}\end{array}\right]$

$=\sigma_{n}^{2}\left[\begin{array}{llll}G_{x x} & G_{x y} & G_{x z} & G_{x b} \\ G_{y x} & G_{y y} & G_{y z} & G_{y b} \\ G_{z x} & G_{z y} & G_{z z} & G_{z b} \\ G_{b x} & G_{b y} & G_{b z} & G_{b b}\end{array}\right]$

The matrix elements quantify the satellite geometry.

\section{B. Dilution of Precision}

Dilution of precision is a function of the satellite constellation, more precisely of the geometry between the receiver and satellite. Thus, calculating the DOPs does not require any observations. The DOP values can be predicted using the satellite almanac data or the satellite orbit information. There are several types of DOPs, and each one can be obtained using the diagonal elements of $G$, independently from the others:

- Horizontal DOP (HDOP): measures the indecision in the horizontal position of the navigation solution: 
$H D O P=\frac{\sqrt{\sigma_{x}^{2}+\sigma_{y}^{2}}}{\sigma_{n}}=\sqrt{G_{x x}+G_{y y}}$

- Vertical DOP (VDOP): corresponds to the uncertainty in the vertical position of the navigation solution;

$V D O P=\frac{\sigma_{Z}}{\sigma_{n}}=\sqrt{G_{z z}}$

- Position DOP (PDOP): represents the uncertainty in the spatial (3D) position of the navigation solution;

$P D O P=\frac{\sqrt{\sigma_{x}^{2}+\sigma_{y}^{2}+\sigma_{z}^{2}}}{\sigma_{n}}$

$=\sqrt{G_{x x}+G_{y y}+G_{z z}}$

- Time DOP (TDOP): stands for the uncertainty of the receiver clock;

$T D O P=\frac{\sigma_{b}}{\sigma_{n}}=\sqrt{G_{b b}}$

- Geometric Dilution of Precision (GDOP): is the combination of all the components that define the impact of geometry on the rapport between the measurement error and the position determination error;

$G D O P=\frac{\sqrt{\sigma_{x}^{2}+\sigma_{y}^{2}+\sigma_{z}^{2}+\sigma_{b}^{2}}}{\sigma_{n}}=\sqrt{G_{x x}+G_{y y}+G_{z z}+G_{b b}}$

- Thus DOP terms are interconnected by the following relationship:

$\mathrm{PDOP}^{2}=\mathrm{HDOP}^{2}+\mathrm{VDOP}^{2}$

$\mathrm{GDOP}^{2}=\mathrm{PDOP}^{2}+\mathrm{TDOP}^{2}$

GDOP is the result of $1 / \mathrm{Vol}$ where $\mathrm{Vol}$ is the volume of a tetrahedron that is formed linking the receiver position and the four satellites. The best geometry to achieve the highest accuracy for point positioning is when the volume of the tetrahedron is a maximum: this situation needs a minimum value of GDOP [16].

An ideal distribution for four satellites consists of one above the receiver, while the remaining three are separated from each other by $120^{\circ}$ in azimuth near the horizon. For this situation, the GDOP value is near to 1 .

GDOP is widely used because it is easy to calculate, based on four satellite observations and invariant as the mathematical DOP [17]. Today GDOP is a standard satellite choice because it plays an important role in determining the accuracy of the positioning [18]. All receivers use algorithms that are able to select a subset of at least four satellites with the minimum GDOP.

Even if the minimum number of satellites required to position and estimate clock off-set is four, usually more than four satellites are selected to increase the estimation robustness and to reduce the degradation in the estimate accuracy that is introduced using subsets [19]. This approach is frequently adopted for multi-GNSS constellation.

\section{APPLICATION}

\section{A. Adopted Approach}

The aim of this paper was to calculate a new index, named the Temporal Variability of Geometric Dilution of Precision (TVGDOP), in order to determine the variability in the visibility of satellites in a defined period of time under different scenarios. Simulation tests were performed on a station, located in Naples (Italy). This station is referred to UTM/WGS84 and its coordinates are: $\varphi=40^{\circ} 52^{\prime}, 27^{\prime}$ '.05 and $\lambda=14^{\circ} 17^{\prime} 27^{\prime \prime} .79$ (440263.095, 4525031.994 meters), and it is 81.47 meters above the mean sea level (Fig. 5).

Trimble Planning [20] was used to calculate the GDOP related to date and time. The Ephemeris file containing the orbital parameters concerning each satellite of the considered positioning systems, was downloaded onto the program to evaluate GDOP values.

Our approach is based on three different combinations of positioning systems, with measurements based on:

- GPS;

- GLONASS;

- GPS and GLONASS.

For the above three solutions, satellites availability and GDOP were calculated considering intervals of 15 minutes, for a total of 96 daily samples. The simulations covered a period of 32 days (January 25 - February 25, 2016). To render the simulation more realistic, particular attention was reserved to cut-off angle, the altitude below which a satellite is no visible because obstructed by terrain morphology. Variable values were assumed for cut-off angle: $10^{\circ}, 15^{\circ}, 20^{\circ}, 25^{\circ}, 30^{\circ}$.

Elevation Cut-off excludes from the calculations all satellites below that height on the horizon. Thus a compensation for the obstruction of those satellites by each specific morphological situation is introduced, i.e. there is assumed to be a clear view of the sky above the elevation cutoff, which means a conic space of satellite visibility. In real surveys, particular obstacles such as neighbouring buildings and trees or mountains (Fig. 6) can mask the GNSS satellites.

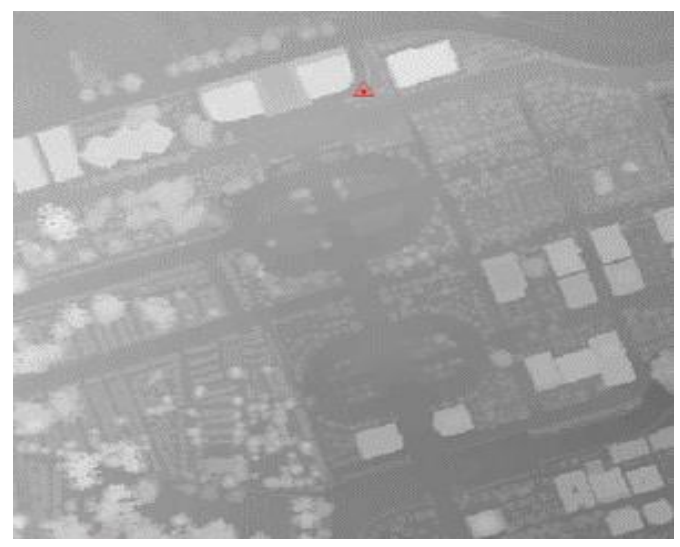

Fig. 5. Station location on the map built from DSM. 


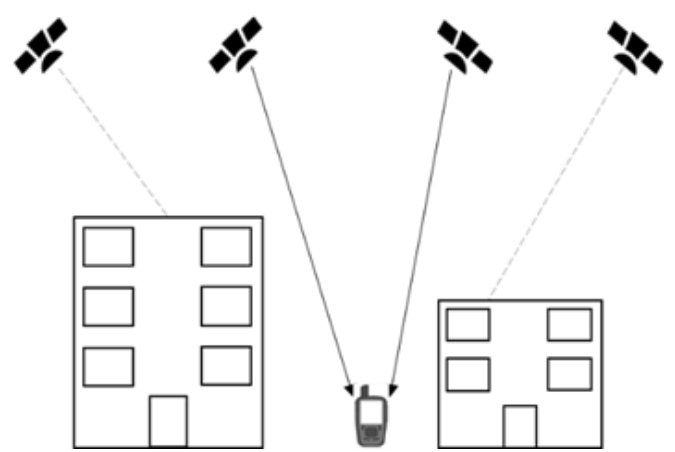

Fig. 6. Example of obstructed view.

Subsequent obstacles that appear along the horizon at the selected location for GNSS measurements are considered. Consequently, another case is investigated, with the same station and the same dates, but with the introduction of an obstruction scenario.

Trimble Planning enables obstacles that appear along the horizon to be drawn using the Obstruction Editor at the location where the GNSS measurements are taken. These include not only the local terrain, but also buildings such as houses or bridges and natural components such as trees. This may be entail reading topographic maps and/or surveying the area. A different and faster approach to define the obstacles caused by the territorial morphology is to use a DSM (Digital Surface Model). A DSM supplies the highest points within a defined grid box [21]. This can be obtained using airborne laser scanning. It is the result of the first echo the laser receives for each laser pulse sent out, and represents the tops of buildings, trees, and other objects, or the ground, if unobstructed [22]. As with DEMs (Digital Elevation Models) [23], a DSM is usually supplied like a grid, i.e. a sample of heights for a number of points that are spaced in regular way. A DTM (Digital Terrain Model) reports the variability of the ground elevation, whereas a DSM depicts the elevation of the top surfaces of buildings, trees, towers and other features elevated above the bare earth [24]. Fig. 7 shows a comparison of a DSM and DTM.

A detailed DSM of the considered area supplied by the local government administration of Naples as a grid interpolation of LIDAR data with cell resolution $1 \mathrm{~m}$ was used initially in MicroDEM [25], a free program that works with elevation plots as a function of position. By selecting the position on the map where the GNSS measurements will be taken, the following options are set:

- Max Horizon: How far out it is possible to see, in meters. The Max Horizon will range not beyond than the borders of the DEM.

- Radial precision: Resolution for drawing the radial distances from the considered position as far as the horizon. A shorter distance is more precise, but more time is necessary.

- Angular precision: The misure of the angle between subsequent radials; for this application, 1 degree is set;
- Observer above ground: The vertical distance between the observer and the ground; in this case, the height of the GNSS unit (or its antenna) above the ground.

- Horizon: the characteristics (thickness and color) of the line thar represents the limit of the horizon.

The horizon blocking line is drawn on the map derived from the DSM. The user position is represented by a red square while the horizon blocking line fixes the limit to his field of view (Fig. 8).

The software creates three graphs:

- The first graph (Fig. 9) shows the altitude (the vertical angle) related to the azimuth;

- The second graph (Fig. 10) shows the distance from the user's position to the topographic horizon related to the azimuth;

- The third graph (Fig. 11) illustrates the line of topographic horizon considering a 360 degree wiew.

MicroDEM exports vertical angle values as a function of the azimuth as a text file that can be read by Trimble Planning software. Given with the user's position, the Obstruction Editor plots the text file to detect the occurrence of significant obstacles during the survey. Once the parameters have been set, it is possible to calculate accurate DOPs for the chosen location in relation or not to the obstacles. The flowchart of our approach is reported in Fig. 12.

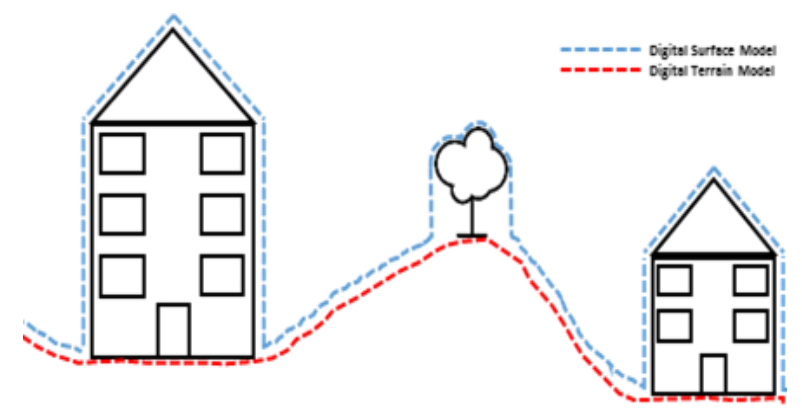

Fig. 7. Examples of a Digital Surface Model and a Digital Terrain Model.

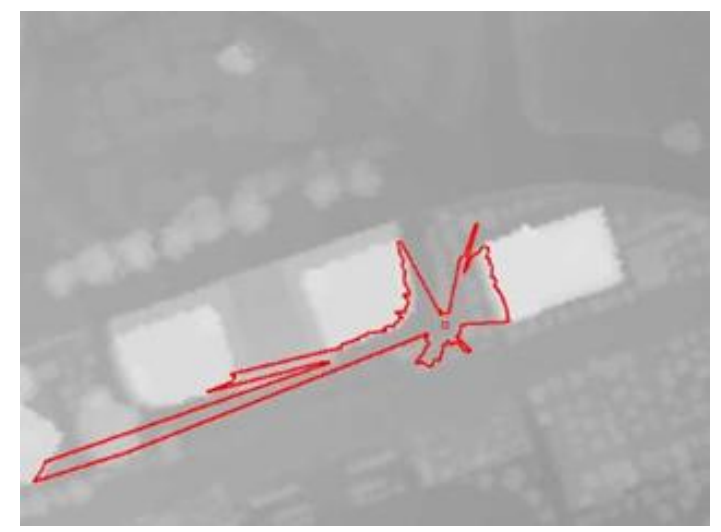

Fig. 8. The Horizon Blocked Area on the Map for the Observer Point. 


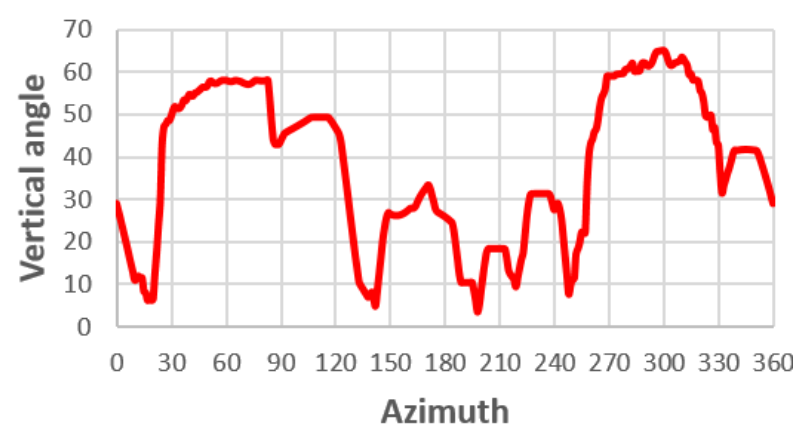

Fig. 9. Representation of Altitude as a Function of the Azimuth.

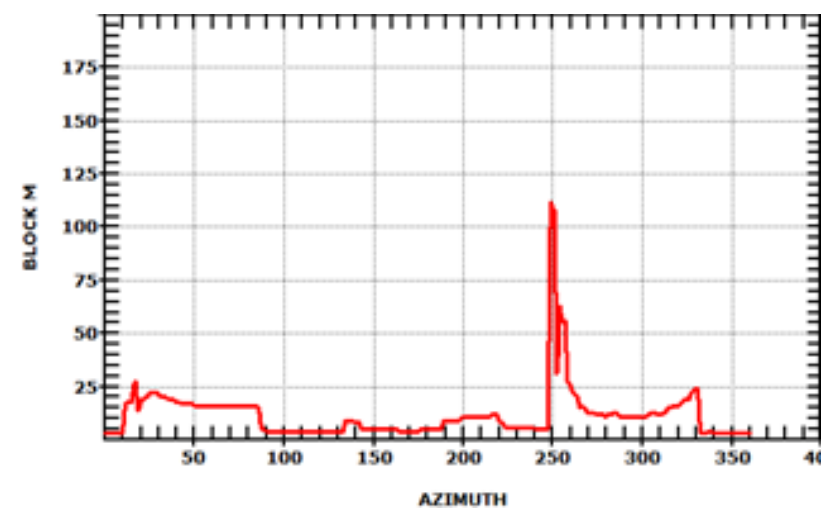

Fig. 10. Representation of Distance from the user's Position to the Topographic Horizon as a Function of the Azimuth.

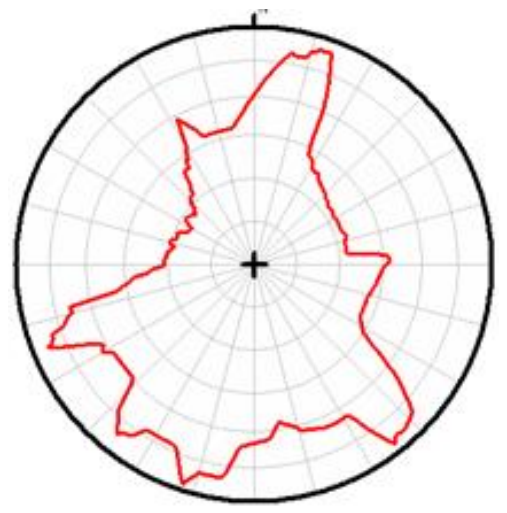

Fig. 11. Topographical Map Shows Area where Blockage Horizon Occurs.

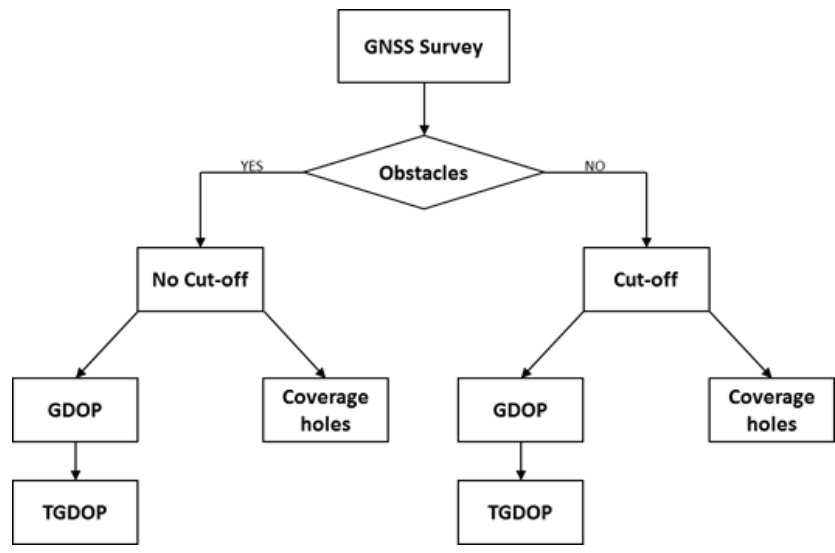

Fig. 12. Flowchart of the Adopted Approach.
In order to determine the GDOP, it is first necessary to decide whether to consider the real obstacle. If they are taken into account, the GDOP calculation method does not consider cut-off angles. If the real obstacles are not contemplated, obstruction scenarios with different elevation cutoff values are studied.

In all cases, the considered period of time is 32 days: for every satellite configuration (GPS, GLONASS, GPS+GLONASS), a minimum collection of available satellites is necessary to achieve reliable GDOP values. More precisely, four satellites for single constellations and five for the GNSS combination are required. For the GNSS, a system time difference parameter is introduced for integrated GPS/GLONASS observation processing [26]. In fact, if the measurements of these different GNSS are combined, the synchronization between the internal GPS and GLONASS receiver clock must be evaluated. Two parameters are contemplated: the unavailability of the minimum number of satellites required for positioning and the temporal variability of GDOP. The unavailability of the minimum number of satellites is determined by the statistical analysis of the daily coverage holes which are the number of times for which less than 4 (for GPS or GLONASS) and 5 (for GPS+GLONASS) satellites are in view during each day. The temporal variability of GDOP is supplied by our proposed index named the Temporal Variability of GDOP:

$T V G D O P=\sum_{i=1}^{N} \frac{\left(\frac{1}{G D O P_{i}}\right)}{N}$

where:

- $\mathrm{N}$ is the number of the intervals included in the considered period of time, for example one day (24 hours, 96 intervals).

The introduction of the inverse of GDOP is necessary to contain all values within the interval $0-1$. In the worst cases, GDOP is high, thus its inverse is small with only a slight contribution to TVGDOP.

\section{B. Results and Discussion}

Tables I and II report the statistics of the coverage holes, the parameter above defined to express (GPS, GLONASS, GPS+GLONASS) satellites visibility, in the absence and presence of obstacles.

The results of Table I highlight how at least four GPS satellites are always visible until the 20 degrees cut-off angle; in fact, coverage holes start to appear after this value although only in small quantities. The GLONASS constellation has the worst performance, presenting coverage anomalies starting at a 15 degrees cut-off. Compared to the GPS, the Russian navigation system has at least twice the number of holes as the corresponding American system. The GPS/GLONASS integration leads to a minimum of five satellites that are continuously in view. 
TABLE I. Statistics of THE DAILy COVERAGE HolEs Without REAL OBSTACLES

\begin{tabular}{|c|c|c|c|c|}
\hline \multirow{2}{*}{ Cut-off } & \multirow{2}{*}{ Constellation } & \multicolumn{3}{|c|}{ Coverage holes } \\
\hline & & Min & Max & Mean \\
\hline \multirow{3}{*}{$0^{\circ}-10^{\circ}$} & $G P S$ & \multirow{3}{*}{\multicolumn{3}{|c|}{0}} \\
\hline & $G L N$ & & & \\
\hline & $G P S+G L N$ & & & \\
\hline \multirow{3}{*}{$15^{\circ}$} & $G P S$ & \multicolumn{3}{|l|}{0} \\
\hline & $G L N$ & 0 & 4 & 2 \\
\hline & $G P S+G L N$ & 0 & & \\
\hline \multirow{3}{*}{$20^{\circ}$} & $G P S$ & \multicolumn{3}{|l|}{0} \\
\hline & $G L N$ & 1 & 9 & 4 \\
\hline & $G P S+G L N$ & \multicolumn{3}{|l|}{0} \\
\hline \multirow{3}{*}{$25^{\circ}$} & $G P S$ & 1 & 3 & 2 \\
\hline & $G L N$ & 4 & 12 & 8 \\
\hline & $G P S+G L N$ & \multicolumn{3}{|l|}{0} \\
\hline \multirow{3}{*}{$30^{\circ}$} & $G P S$ & 3 & 9 & 6 \\
\hline & $G L N$ & 10 & 23 & 15 \\
\hline & $G P S+G L N$ & \multicolumn{3}{|l|}{0} \\
\hline
\end{tabular}

TABLE II. Statistics of the Daily Coverage Holes with Real OBSTACLES

\begin{tabular}{|l|l|l|l|}
\hline & Min & Max & Mean \\
\hline$G P S$ & 40 & 51 & 44 \\
\hline$G L N$ & 64 & 78 & 70 \\
\hline$G P S+G L N$ & 2 & 8 & 5 \\
\hline
\end{tabular}

The statistics in Table II highlight that the obstacle incidence is particularly high with the single constellations. GPS has a minimum daily coverage holes $(\mathrm{CHs})$ value four times higher than its corresponding maximum value without obstacles and with a 30 degrees cut-off angle. GLONASS reveals a worse performance: its mean $\mathrm{CHs}$ value (70) is too high considering the total number of daily measurements (96). GPS+GLONASS does not suffer much from this handicap because it has a maximum $\mathrm{CH}$ value equal to $8 \%$ of the daily observations.

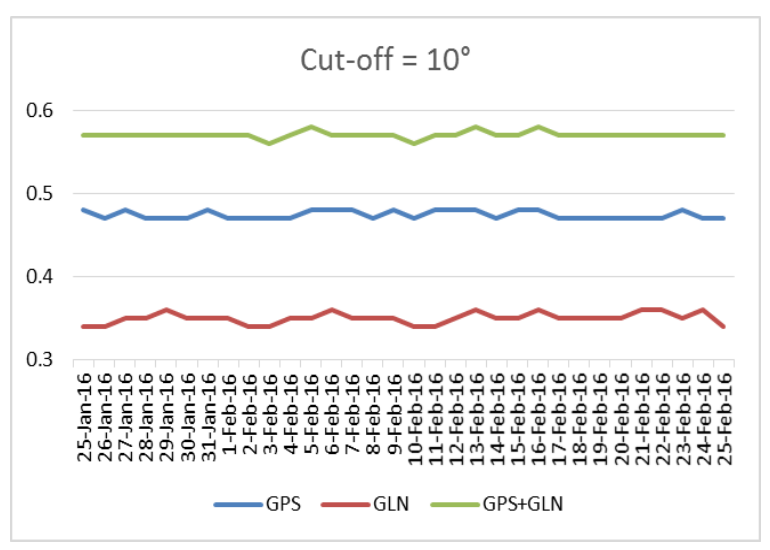

Fig. 13. Daily TVGDOP Values of the Three Constellation Configurations for 10 Degrees Cut-Off.
In order to compare the simulated positioning accuracy of GPS, GLONASS and GPS+GLONASS observations, daily TVGDOP means for the two considered cases (with and without real obstacles), were calculated (Fig. 13 to 18).

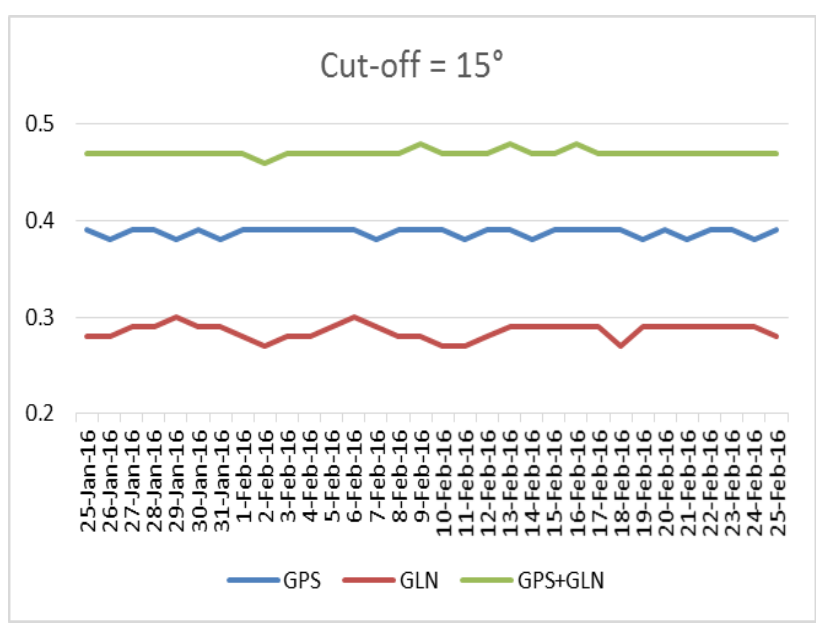

Fig. 14. Daily TVGDOP Values of the Three Constellation Configurations for 15 Degrees Cut-Off.

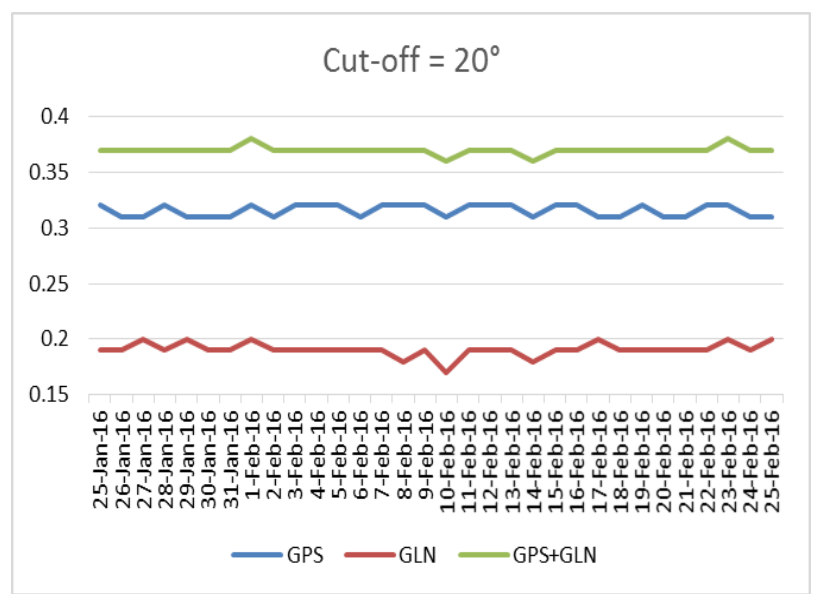

Fig. 15. Daily TVGDOP Values of the Three Constellation Configurations for 20 Degrees Cut-Off.

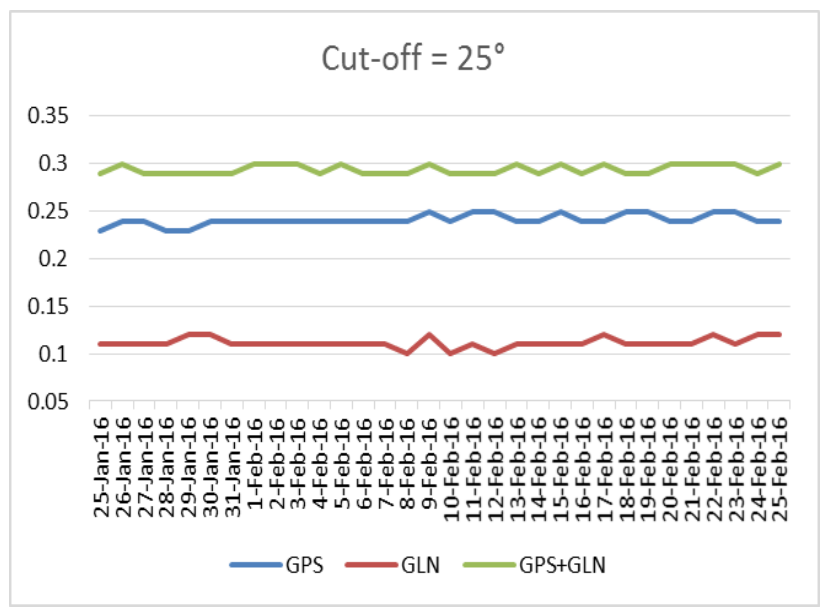

Fig. 16. Daily TVGDOP Values of the Three Constellation Configurations for 25 Degrees Cut-Off. 


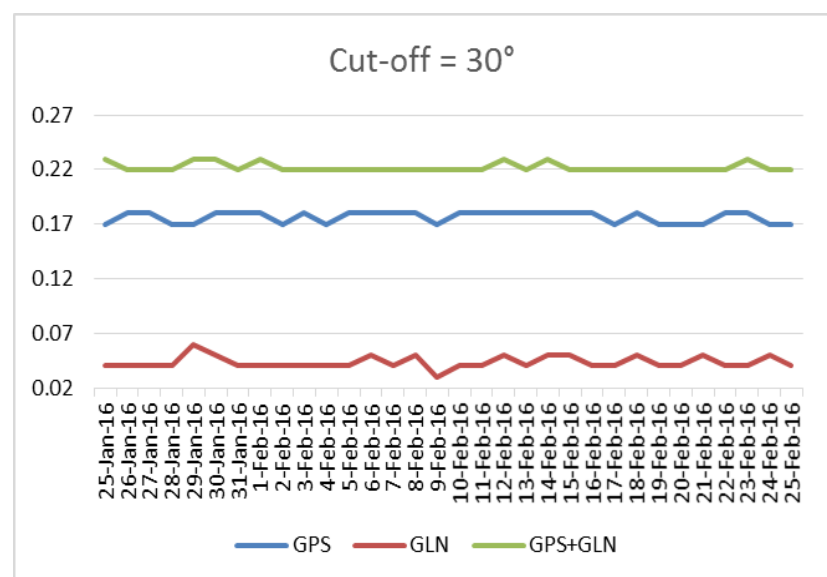

Fig. 17. Daily TVGDOP Values of the Three Constellation Configurations for 30 Degrees Cut-Off.

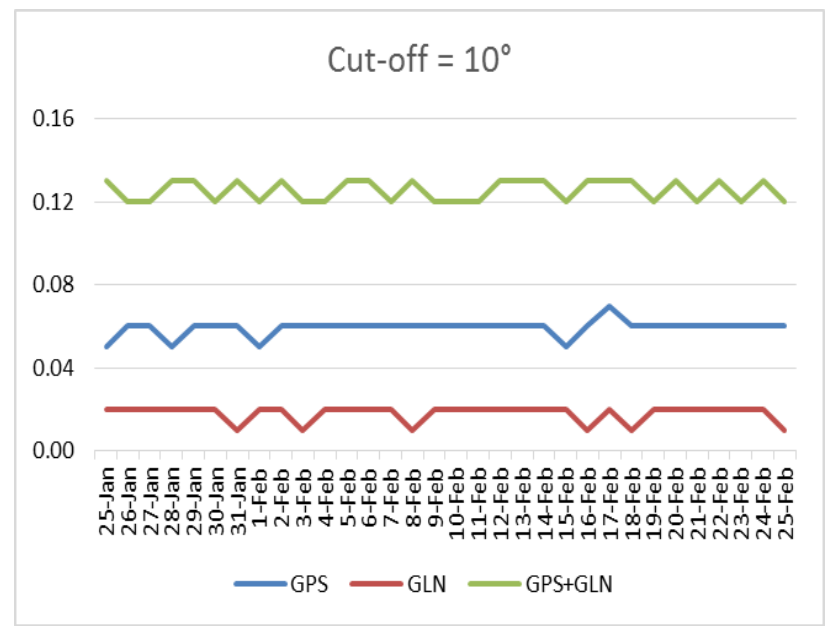

Fig. 18. Daily TVGDOP Values of the Three Constellation Configurations with Real Obstacles.

An increase in a cut-off angle clearly corresponds to a decrease in TVGDOP (Table III).

The index, for the three combinations, is included between the following values:

- 0.18 - 0.47 for GPS;

- 0.04 - 0.35 for GLONASS;

- $0.22-0.57$ for GPS+GLONASS.

In the presence of obstacles, TVGDOP values decrease: GPS+GLONASS combination only has a value which is approximately $28 \%$ of the corresponding case without impediments. However, the impact of the integration is particularly evident because the TVGDOP for GPS+GLONASS presents an increase of $117 \%$ compared with GPS.

In all cases, the proposed index well demonstrates that the integration of GPS and GLONASS produces benefits on satellite geometry as it is expected [27] [28] [29].
TABLE III. STATISTICS OF THE DAILY TVGDOP MEAN VALUES

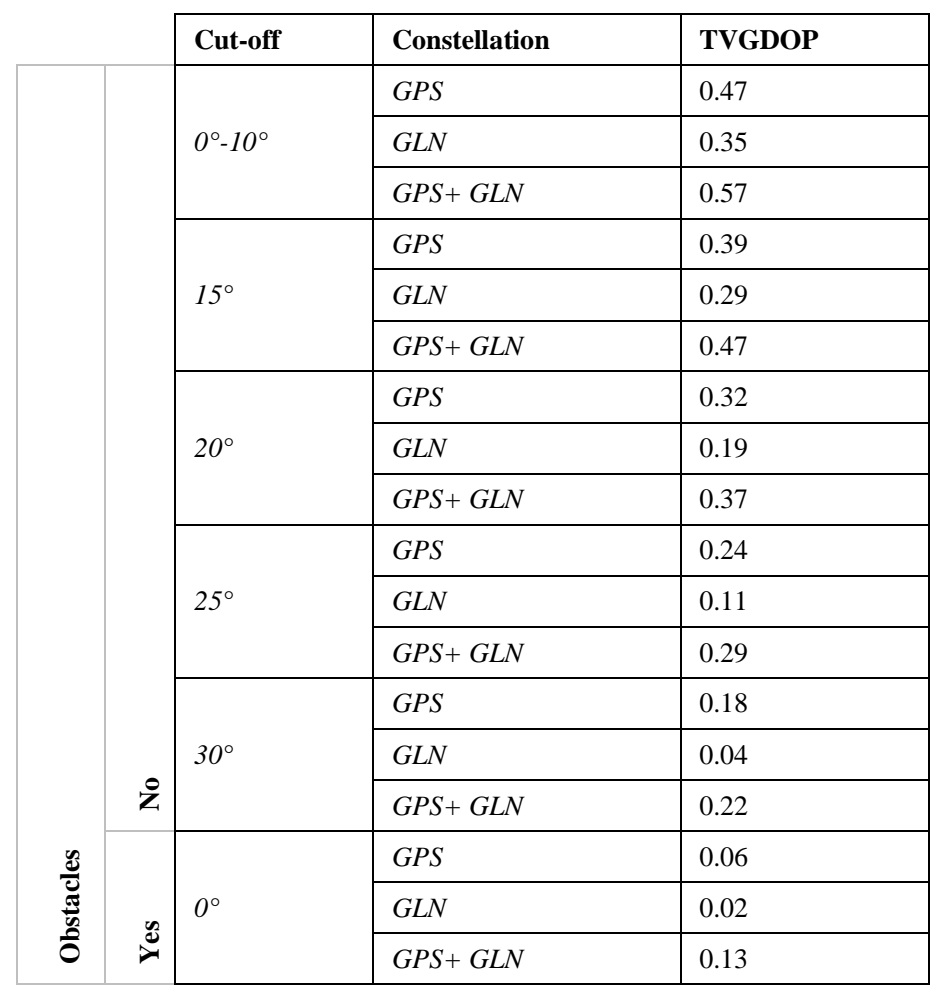

IV. CONCLUSION

This paper aimed to quantify the impact of the integration of GPS and GLONASS systems on satellite geometry. It presents the latest results obtained within a project carried out at Department of Sciences and Technologies (DiST) of the University of Naples "Parthenope".

Using the Ephemeris of both constellations for a long period (32 days), the combination of GPS+GLONASS had not only increased the number of visible satellites, but also optimized their space distribution compared to GPS and GLONASS alone. These positive effects were confirmed by the limited or null unavailability of the minimum number of satellites required for positioning and lower values of GDOP, respectively.

To facilitate a comparison between each single system and their integration, we proposed a new index named TVGDOP, which measures the quality of the satellite geometry with reference to a precise time interval (e.g. one day, one week, etc.). The analysis was carried out for different scenarios, in the absence (but with different cut-off angles) and presence (considering DSM) of real obstacles.

The benefits of GPS+GLONASS were more evident in obstructed spaces such as urban environments where a notable increase in TVGDOP was registered compared to a single constellation.

\section{ACKNOWLEDGMENT}

The research is supported by University of Naples "Parthenope". 


\section{REFERENCES}

[1] S. Dawoud, GNSS principles and comparison, Potsdam University, 2011.

[2] A. K. Maini, and V. Agrawal, Satellite technology: principles and applications, John Wiley \& Sons, 2011.

[3] S.R. Babu, S.I. Dutt, R. Goswami, C.U. Kumari, G.S.B. Rao, and S.S. Rani, "Investigation of GDOP for precise user position computation with all satellites in view and optimum four satellite configuration," Journal of Indian Geophysical Union, vol.13(3), , pp. 139-148, 2009.

[4] C. Cai, and Y. Gao, "Precise point positioning using combined GPS and GLONASS observations," Journal of Global Positioning Systems. vol.6(1), pp. 13-22, 2007.

[5] A. Constantinescu, and R.J. Landry, "GPS/Galileo/GLONASS hybrid satellite constellation simulator-GPS constellation validation analysis," The Institute of Navigation 61st Annual Meeting, pp. 733-737, 2005.

[6] J. Guo, M. Li, X. Li, L. Qu, X. Su, and Q. Zhao, "Precise point positioning with the BeiDou navigation satellite system," Sensors.; 14(1), pp. 927-943, 2014.

[7] Z. Jun, Z. Miaoyan, and Q. Yong, "Satellite selection for multiconstellation," Position, Location and Navigation Symposium, pp. 10531059, 2008.

[8] H.A. Karimi, and D. Roongpiboonsopit, "A multi-constellations satellite selection algorithm for integrated global navigation satellite systems," Journal of Intelligent Transportation Systems. vol.13(3), pp. 127-141, 2009 .

[9] P. Mattos, "Consumer GPS/GLONASS Accuracy and Availability Trials of a One-Chip Receiver in Obstructed Environments," GPS World, vol.22(12), pp. 32-37, 2011.

[10] M. Fritsche, X. Li, X. Ren, H. Schuh, J. Wickert, and X. Zhang, "Precise positioning with current multi-constellation global navigation satellite systems: GPS, GLONASS, Galileo and BeiDou," Scientific Reports, vol.5(8328), pp. 1-14, 2015.

[11] Government B. Ashman, B., 2020. An Introduction to Global Navigation Satellite Systems, NASA Report GSFC-E-DAA-TN76837, 2019.

[12] L. Zhao, L., VP. áclavovic, and J. Douša, J., "Performance Evaluation of Troposphere Estimated from Galileo-Only Multi-Frequency Observations," Remote Sensing, 12(3), p.373, 2020.

[13] R.B. Langley, "Dilution of Precision," GPS World, vol.10(5), , pp. 5259, 1999.

[14] J.B.Y. Tsui, Fundamentals of global positioning system receivers, Wiley-Interscience, 2000.

[15] R.G. Brown, P.Y.C Hwang, Introduction to Random Signals and Applied Kalman Filtering, John Wiley and Sons, 1992.
[16] C. Rizos, "Multi-constellation GNSS/RNSS from the perspective of high accuracy users in Australia," Journal of spatial science, vol. 53(2), pp. 29-63, 2008.

[17] A. Krauter, "Role of the Geometry in GPS Positioning," Periodica Polytechnica: Civil Engineering, vol.43(1), pp. 43-53, 1999.

[18] G. Fan, D. Song, C. Xu, and P. Zhang, "An Algorithm of Selecting more than Four Satellites from GNSS," International Conference on Advanced Computer Science and Electronics Information (ICACSEI 2013), Atlantis Press, pp. 134-138, 2013.

[19] J. Zhang, and M. Zhang, "A fast satellite selection algorithm: beyond four satellites," IEEE Journal of Selected Topics in Signal Processing, vol.3(5), pp. 740-747, 2009.

[20] Trimble, Trimble Planning Software, Sunnyvale, USA, 2019.

[21] C. Eberhöfer, M. Hollaus, W. Karel, and W. Wagner, "Accuracy of large-scale canopy heights derived from LiDAR data under operational constraints in a complex alpine environment," ISPRS Journal of Photogrammetry and Remote Sensing, vol.60(5), pp. 323-338, 2006.

[22] R. Behrendt, "Introduction to LiDAR and forestry, part 1: a powerful new 3D tool for resource managers," The forestry source, vol.17(10), pp. 14-15, 2012.

[23] E. Alcaras, C. Parente, and A. Vallario, "A Comparison of different interpolation methods for DEM production", International Journal of Advanced Trends in Computer Science and Engineering. Vol. 6, pp. 1654- 1659, 2019.

[24] H.K. Heidemann, Lidar base specification - Techniques and Methods, U.S. Geological Survey, 2014.

[25] P. Guth, MICRODEM software, Oceanography Department, U.S. Naval Academy, 2017.

[26] K. Fischer, H. Habrich, and P. Neumaier, "GLONASS Data Analysis for IGS," Proceedings of IGS Workshop and Symposium, University of Berne, 2004.

[27] C. Meneghini, and C. Parente, "Advantages of multi GNSS constellation: GDOP analysis for GPS GLONASS and Galileo combinations," International Journal of Engineering and Technology Innovation, 7(1), pp. 01-10, 2017.

[28] C. O'Driscoll, G. Lachapelle, and M. E. Tamazin, "Investigation of the benefits of combined GPS/GLONASS receivers in urban environments," Proceeding on RIN NAV10 Conference on Position, Location, Timing: Everyone, Everything, Everywhere, 2010.

[29] D. Mortari, J. J. Davis, A.Owis, and H. Dwidar, "Reliable Global Navigation System using Flower Constellation," (IJACSA) International Journal of Advanced Computer Science and Applications, Vol. 4, No. 2, Feb. 2013. 